\title{
MS11-P04 | COMBINING THE USE OF LIBRARIES FROM SEVERAL DISTANT HOMOLOGS IN ARCIMBOLDO_SHREDDER SPHERES
}

Jiménez-Mellado, Elisabet (IBMB - CSIC, Barcelona, ESP); Millán Nebot, Claudia Lucía (Institut de Biologia Molecular de Barcelona, Barcelona, ESP); Medina, Ana (IBMB - CSIC, Barcelona, ESP); Sammito, Massimo (Cambridge Institute for Medical Research, University of Cambridge, Cambridge, GBR); Usón, Isabel (IBMB - CSIC, Barcelona, ESP)

ARCIMBOLDO [1] is a software that exploits secondary and tertiary structure constraints for phasing by using fragments such as ideal polyalanine alpha helices or libraries of local folds derived from structures deposited in the PDB [2]. ARCIMBOLDO combines the location of such small, accurate models with PHASER [3] followed by density modification and auto tracing with SHELXE [4].

ARCIMBOLDO_SHREDDER [5] produces fragment models from homologs whose deviation to the final structure is too large for successful location. Models are cut in equivalent volumes respecting structural units and annotated into separate rigid groups with ALEPH [6]. Gyre and gimble refinement enhance the convergence of the method, improving starting models with large deviations from the target.

This work explores the combination of model fragments from several distant homologs within the SHREDDER program, collaborating with SBGrid [7]-supported high-performance computing resources to overcome the massive scale computing requirements needed. Our goal is to be able to exploit the maximum amount of prior information about our unknown fold and to evaluate if we get larger discrimination by the use of multiple homologs.

[1] Millán et al., (2015) IUCrJ. 1, 95.

[2] Burley et al. (2019) Nucleic Acids Research 47: D464

[3] McCoy et al. (2007) Appl Crystallogr. 40, 658

[4] Usón \& Sheldrick (2018) Acta Crystallogr. D74, 106

[5] Millán et al. (2018) Acta Crystallogr D 74: 290

[6] Medina et al. (2019) In preparation

[7] Morin et al. (2013) eLife. 2: e01456 